\title{
Babesia microti acquired in Canada
}

\author{
Jiayu Yang MD, Catherine Smith MD, Anthony Battad MD MSc MPH
}

Cite as: CMAJ 2021 August 9;193:E1213-7. doi: 10.1503/cmaj.201983

西

n August 2019, a 75-year-old man presented to the emergency department in Winnipeg with a 6-week history of fever, myalgia, lethargy and $7-\mathrm{kg}$ weight loss. After 3 weeks of symptoms, he had visited his family physician, who prescribed a 1-week course of amoxicillin-clavulanic acid. When the symptoms persisted despite antibiotic treatment, he went to a community emergency department, where the physician identified anemia and thrombocytopenia (Table 1). After this, an urgent outpatient computed tomography (CT) scan of his chest, abdomen and pelvis was unremarkable. The patient later presented to our emergency department with persistent symptoms, and we admitted him for further investigations.

The patient recalled first feeling ill while boating at Pelican Lake in Ninette, Manitoba, at which time he noticed a new, red, thumb-sized lesion on his right leg. He did not remember finding any ticks or other skin lesions on his body. His travel history was notable for a visit to Arizona 6 months before symptom onset, but he had not been outside North America. Eleven weeks before symptom onset, he had camped at Birds Hill Provincial Park in Manitoba. Seven weeks before his symptoms started, he had visited Burlington, Ontario (Figure 1). He had donated blood 7 weeks before presentation.

\section{KEY POINTS}

- Babesia microti, transmitted by Ixodes scapularis, is the primary cause of babesiosis in North America.

- Babeosis usually presents with intermittent fever, malaise, anemia and thrombocytopenia, and can coexist with other tickborne infections.

- Babesiosis can mimic malaria on blood smear. Thin blood smears and polymerase chain reaction testing are diagnostic.

- The range of I. scapularis has expanded into Canada. Tick-borne diseases, including babesiosis, should be included in the differential diagnosis in Canada, and empiric treatment should be considered.

On admission to hospital, the patient was febrile $\left(38.6^{\circ} \mathrm{C}\right)$ and tachycardic (105 beats/min). His physical examination was unremarkable, with no insect bites, rashes, lymphadenopathy, hepatosplenomegaly, or jaundice. Given his recent negative CT scan and nonspecific symptoms, the likely cause was infectious, and our differential diagnosis focused on viral and atypical infections.

Investigations showed that the patient's pancytopenia had progressed since his last emergency department visit, and his

Table 1: Laboratory investigation results in a 75-year-old man with babesiosis

\begin{tabular}{|c|c|c|c|c|}
\hline Test & $\begin{array}{l}1 \text { week before } \\
\text { admission }\end{array}$ & On admission & $\begin{array}{l}\text { 1-week after } \\
\text { discharge }\end{array}$ & Reference \\
\hline Hemoglobin (g/L) & 119 & 95 & 95 & $140-180$ \\
\hline Platelet count $\left(\times 10^{9} / \mathrm{L}\right)$ & 265 & 67 & 283 & $140-440$ \\
\hline Urea (mmol/L) & 7.9 & 11.2 & 6.0 & $2.8-7.1$ \\
\hline Aspartate aminotransferase (U/L) & 28 & 81 & 28 & $10-32$ \\
\hline Alanine aminotransferase (U/L) & 24 & 66 & 39 & $<30$ \\
\hline Alkaline phosphatase (U/L) & & 79 & & $30-120$ \\
\hline Y-glutamyl transferase (U/L) & & 54 & 60 & $5-38$ \\
\hline Lactate dehydrogenase (U/L) & & 603 & 79 & $120-230$ \\
\hline International normalized ratio & & 1.1 & & $0.9-1.1$ \\
\hline Haptoglobin (g/L) & & $<0.1$ & & $0.2-2.0$ \\
\hline
\end{tabular}




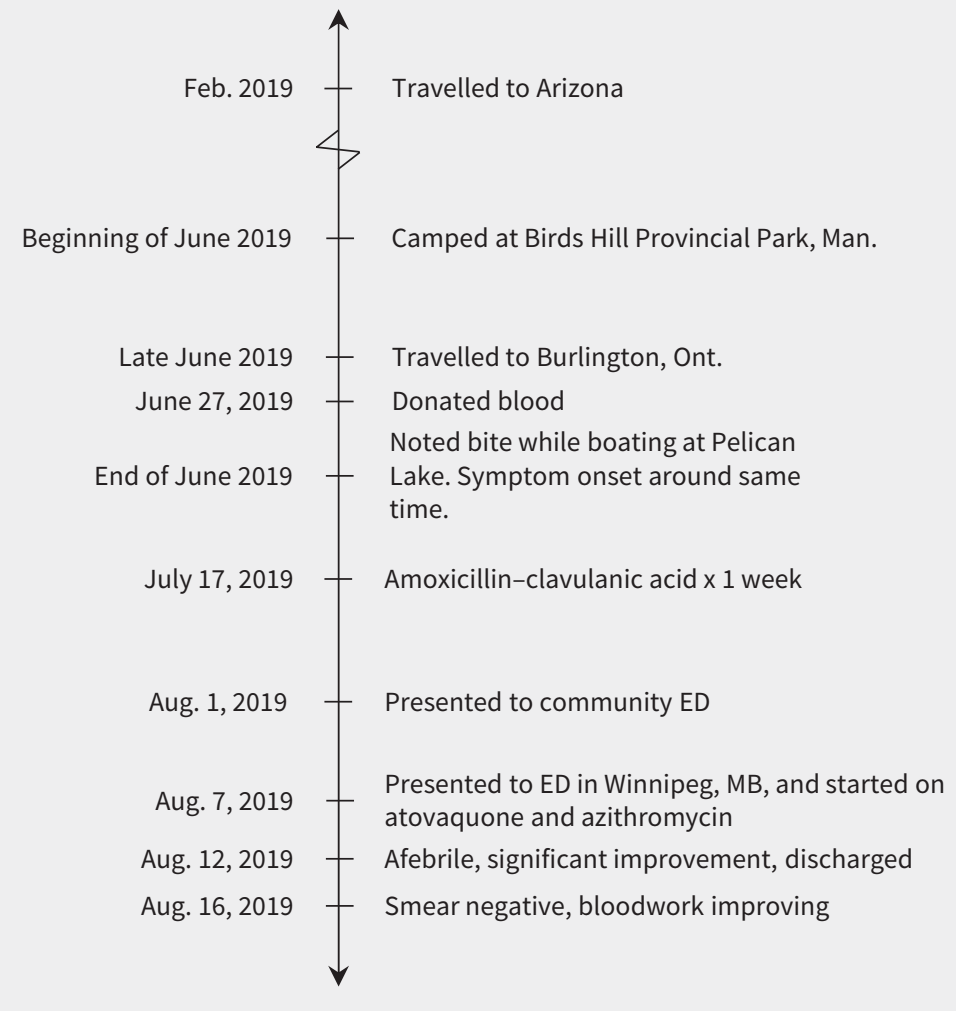

Figure 1: Course of events and clinical course of a 75-year-old man with babesiosis. Note: ED = emergency department.

liver enzyme levels were elevated. Laboratory investigations presented in Table 1 showed evidence of hemolysis and acute kidney injury. Blood culture, urine culture and serology tests for hepatitis A, B and C; HIV; cytomegalovirus; Epstein-Barr virus; West Nile virus; and Borrelia burgdorferi were negative. We did not test for anaplasmosis at the time. His chest radiograph was unremarkable.

To further investigate the patient's anemia and thrombocytopenia, we ordered a peripheral blood smear to rule out microangiopathic hemolytic anemia, which showed intraerythrocytic forms suggestive of malaria. We then ordered thick and thin smears. The initial parasite load was $0.2 \%$, which subsequently peaked at $0.8 \%$. With the patient's history suggestive of an insect bite and no reported travel to a malaria-endemic region, we suspected babesiosis and started empiric treatment with atovaquone and azithromycin. The patient became afebrile by treatment day 3 .

A hematopathologist's further analysis of the peripheral smear supported a diagnosis of babesiosis (Figure 2 and Figure 3), and the infectious cause was ultimately confirmed as Babesia microti by polymerase chain reaction (PCR) test. The patient's parasitemia was reduced to $0.1 \%$ at the time of his discharge 5 days after admission and 0\% 1 week after discharge. His renal function continued to improve, and his pancytopenia and liver enzyme abnormalities resolved (Table 1).

We contacted Canadian Blood Services, which tested the patient's donated blood using PCR, and found it to be positive for B. microti.

\section{Discussion}

\section{Epidemiology of babesiosis in Canada}

Babesiosis is caused by the intraerythrocytic protozoa genus Babesia, primarily transmitted by Ixodes ticks, and occasionally through blood transfusion, organ transplantation and transplacentally. ${ }^{1}$ Although many Babesia species are known to cause infections in humans, most instances of babesiosis in North America are caused by B. microti, which is transmitted by I. scapularis, endemic to the northeastern and northern-midwestern United States, where the incidence of babesiosis is about 2000 cases per year (Figure 4; see also https://www.cdc.gov/parasites/babesiosis/ resources/babesiosis_surveillance_summary_2018.pdf). ${ }^{1}$

Until recently, babesiosis was not found in Canada. A seroprevalence study in donated blood in 2013 showed no $B$. microti antibody-positive samples in about 14000 tested. ${ }^{2}$ However, with climate change, I. scapularis has migrated north, and is now found in Manitoba, Ontario, Quebec, Nova Scotia and New Brunswick. ${ }^{3}$ Another seroprevalence study in 2018 tested for $B$. microti by transcription-mediated amplification (TMA) in 50752 samples and 1 positive result was found (from Manitoba). A randomly selected subset of 14758 TMA-negative samples was tested for Babesia immunoglobulin $G$, with 4 positive results (all from southwestern Ontario). Only 1 out of the 5 donors recalled having flu-like symptoms 1 month after donation. ${ }^{4}$ 


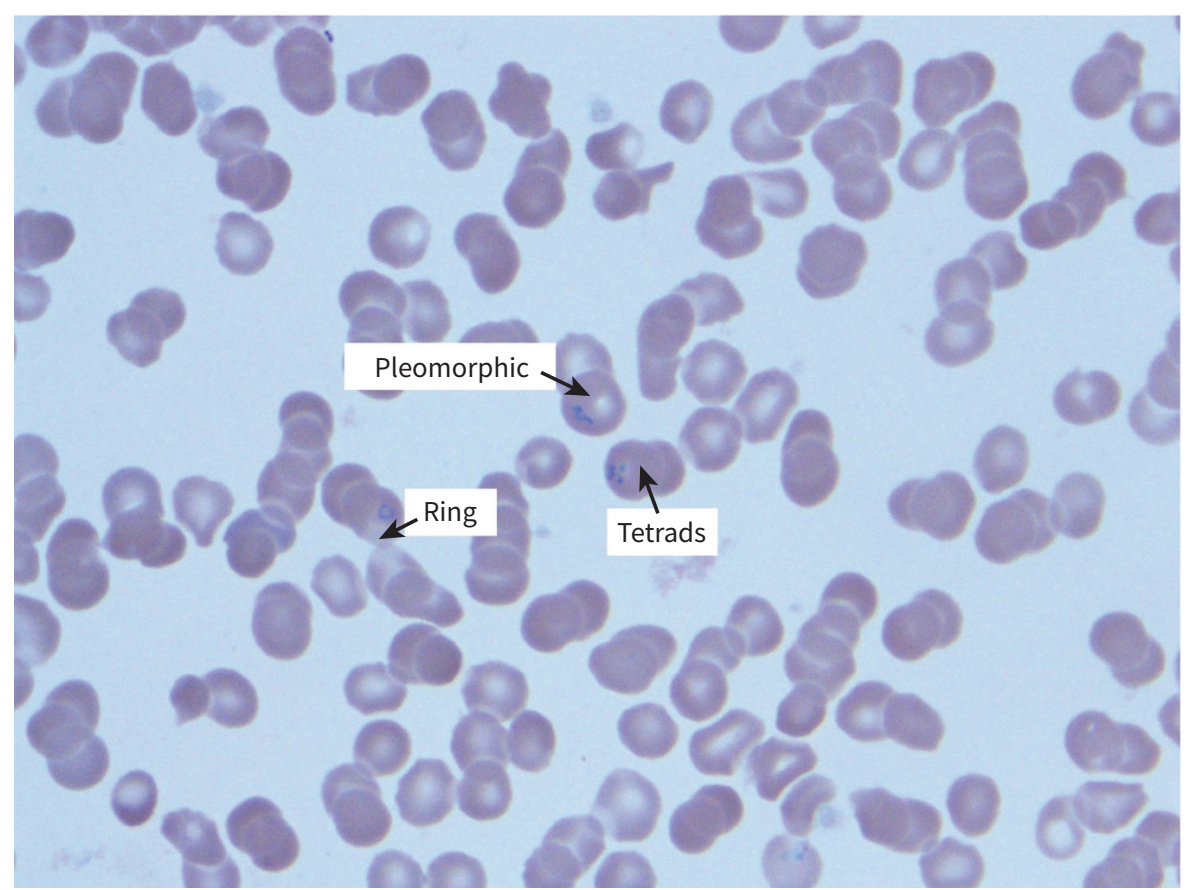

Figure 2: Blood smear in a 75-year-old man with babesiosis, showing pleomorphic and ring trophozoites, and "Maltese cross" tetrad merozoites.

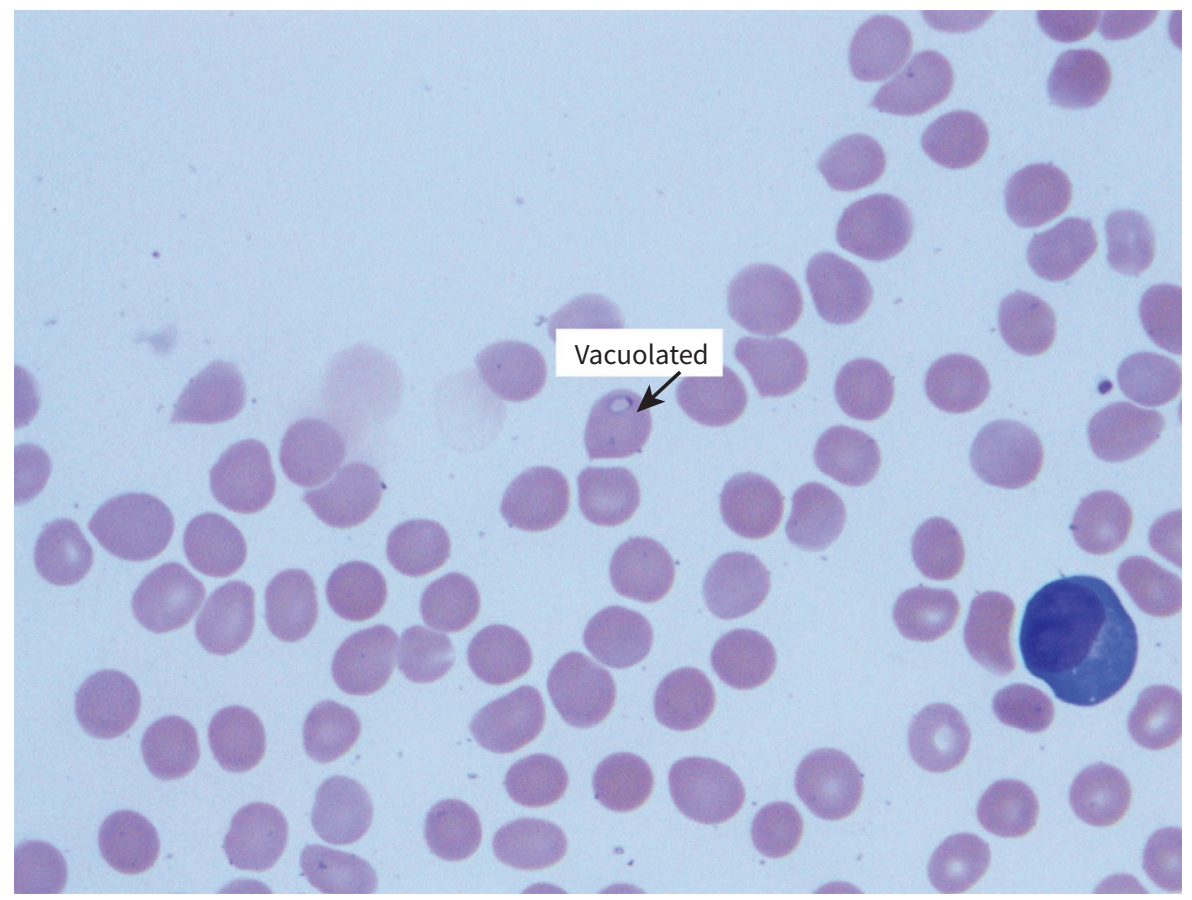

Figure 3: Blood smear in a 75-year-old man with babesiosis, showing vacuolated trophozoites.

\section{Transmission and clinical} presentation

In North America, B. microti is transmitted primarily by the vector I. scapularis from late spring to early autumn. The white-footed mouse, Peromyscus leucopus, is the principal reservoir. Ixodes scapularis feeds on the white-tailed deer, Odocoileus virginianus, which does not become infected, but amplifies the $I$. scapularis population. ${ }^{1}$ The incubation period is typically 1-6 weeks after a tick bite.

Similar to Plasmodium species, Babesia infects erythrocytes, with release of merozoites leading to hemolysis and associated clinical complications. Babesiosis can range from asymptomatic to severe, life-threatening illness. Asymptomatic infection is common, with a prospective 10-year study showing this in about one-quarter of infected adults and half of children. ${ }^{8}$ However, clinical manifestations have been documented in previously asymptomatic patients after splenectomy or immunosuppression. ${ }^{9}$ Fever is a salient feature in babesiosis. Patients typically present with intermittent fever, chills, sweats, headache, myalgia and anorexia, as well as progressive malaise and fatigue. Physical examination and laboratory investigations show features consistent with the pathogenesis, including pallor, jaundice, hepatosplenomegaly, hemolytic anemia, thrombocytopenia, and elevated creatinine and liver enzyme levels. Severe disease often occurs in immunocompromised patients, particularly in those of advanced age, with manifestations of severe anemia including acute respiratory distress syndrome, disseminated intravascular coagulation, congestive heart failure, renal failure, shock and death. Mortality rates as high as $20 \%$ have been reported for severe babesiosis. ${ }^{1}$
The first case of locally acquired babesiosis in Manitoba was documented in 2013, where B. microti was confirmed by PCR in a 7-year-old boy with a complex medical history remarkable for asplenia secondary to midgut malrotation. ${ }^{5}$ Another probable case was reported in Manitoba in 2016, the only Canadian jurisdiction where it is a reportable disease. ${ }^{6}$ Since then, 1 further case of babesiosis from $B$. duncani was found in a 70-year-old man in southern Ontario after a bite from an I. scapularis nymph, in $2017 .{ }^{7}$

\section{Diagnosis}

Babesiosis can be diagnosed on Giemsa-stained or Wrightstained thin blood smears. Most commonly seen are ring forms of the parasite, which are commonly mistaken for Plasmodium falciparum trophozoites. However, Babesia can be distinguished from $P$. falciparum by the form of its merozoites. The parasite is called a sporozoite as it enters the erythrocyte. It develops into a trophozoite inside the cell, which then moults 


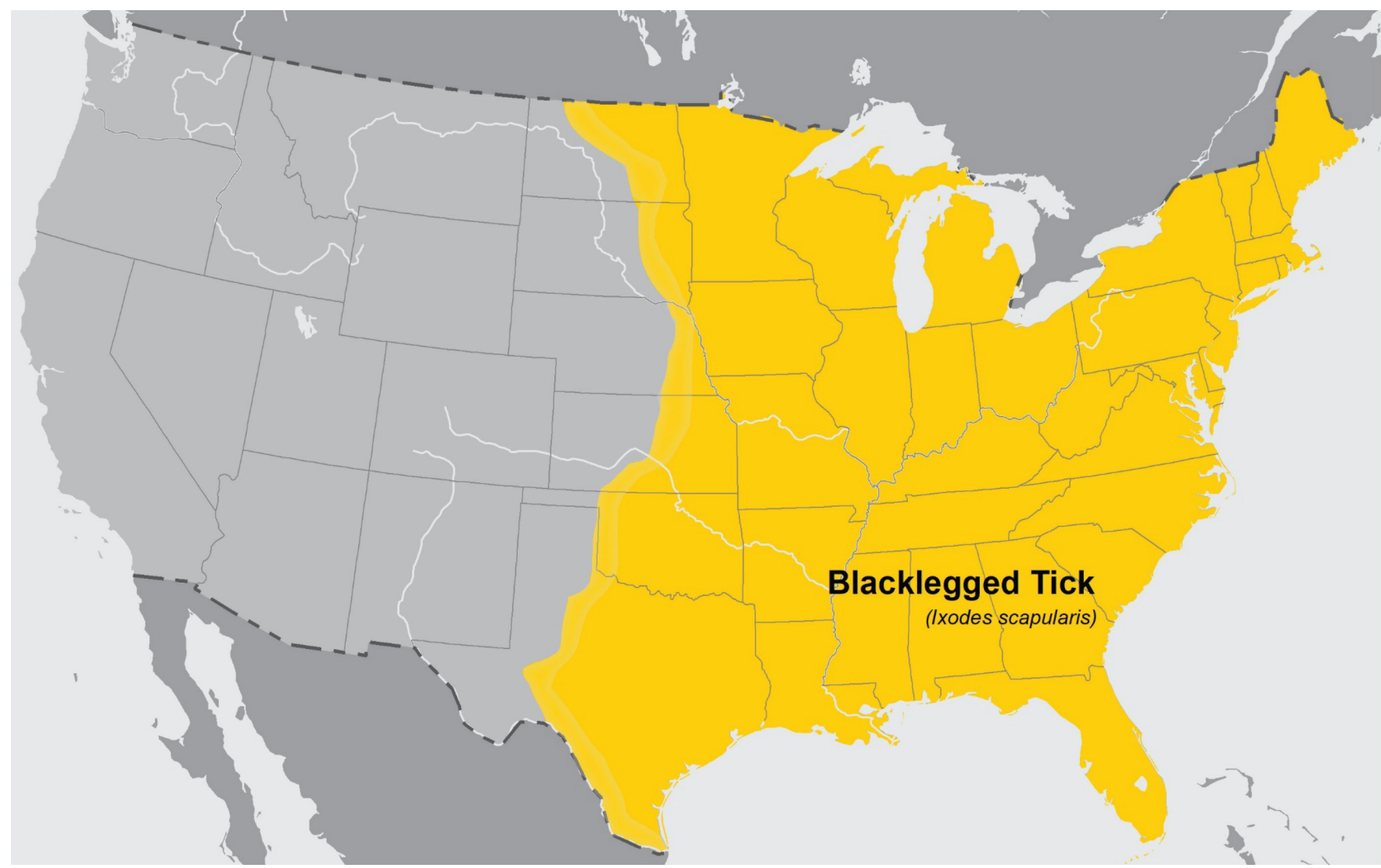

Figure 4: Estimated distribution of Ixodes scapularis (blacklegged tick) in the United States as of 2018. (Image reproduced from the Centers for Disease Control and Prevention [CDC]. This use does not imply endorsement by the CDC. Material otherwise available on CDC website for no charge.)

into merozoites, the tetrad "Maltese cross" formation of which is pathognomonic of the infection (Figure 1).1,10 Polymerase chain reaction is a more sensitive test than a blood smear, and can identify the specific Babesia species in addition to diagnosing the infection. ${ }^{1,10}$ Babesia serology can also assist in diagnosis, with an antibody titre level of 1:1024 or greater suggestive of a recent or active infection, although confirmatory evidence requires a fourfold increase between acute and convalescent sera. ${ }^{1,10}$

I. scapularis is known to transmit other pathogens, including Borrelia species, Anaplasma phagocytophilum, Ehrlichia species and Powassan virus. A 2016 review found that coinfections occur in 1\%-28\% of Ixodes ticks in Lyme diseaseendemic areas in North America. ${ }^{11}$ The most frequently documented coinfection is $B$. burgdorferi and $B$. microti, occurring in $6 \%-23 \%$ of patients with babesiosis. ${ }^{11}$ Therefore, it is important to investigate for potential coinfection in patients with babesiosis.

\section{Treatment}

The recommended treatment for babesiosis is atovaquone $750 \mathrm{mg}$ taken orally every 12 hours, combined with azithromycin 500-1000 mg orally, followed by $250-500 \mathrm{mg}$ orally daily for 7-10 days. An alternative regimen is clindamycin 300-600 mg taken intravenously every 6 hours or $600 \mathrm{mg}$ orally every 8 hours, and quinine $650 \mathrm{mg}$ orally every $6-8$ hours,,$^{10}$ which is associated with several adverse effects, but is recommended for severe disease. ${ }^{1}$ In immunocompromised patients, the duration of treatment should be extended beyond 6 weeks, including at least 2 weeks after a negative smear. ${ }^{10}$ In patients with severe babesiosis with parasitemia $\geq 10 \%$, severe hemolysis, or pulmonary, renal or hepatic impairment, exchange transfusion should be considered. ${ }^{10}$

\section{Conclusion}

We presented a case of $B$. microti in an immunocompetent adult, most likely acquired in Manitoba, Canada, given the typical 1- to 6-week incubation period, although the possibility remains that infection was acquired in Ontario. It is unlikely that our patient was infected on his prior trip to Arizona, as I. scapularis is not endemic to the region and, although a prolonged incubation period is possible, it would not be typical. With climate change and expansion in the range of $I$. scapularis, the incidence of tick-borne diseases, including babesiosis, is rising in Canada. ${ }^{2,3}$ It is thus crucial to include babesiosis as part of the differential diagnosis in patients presenting with fever, particularly in the summer, even if no travel outside Canada is reported. Detection of intraerythrocytic parasite forms in the absence of travel to a malaria-endemic region should raise suspicion of babesiosis and prompt consideration of empiric treatment. Mandatory reporting to public health authorities should 
be considered in all jurisdictions across Canada, to improve documentation of cases, track prevalence and increase awareness in the health care community.

\section{References}

1. Krause PJ. Human babesiosis. Int J Parasitol 2019;49:165-74.

2. O'Brien SF, Delage G, Scalia V, et al. Seroprevalence of Babesia microti infection in Canadian blood donors. Transfusion 2016;56:237-43.

3. Bouchard C, Dibernardo A, Koffi J, et al. Increased risk of tick-borne diseases with climate and environmental changes. Can Commun Dis Rep 2019;45:83-9.

4. Tonnetti L, O'Brien SF, Gregoire Y, et al. Prevalence of Babesia in Canadian blood donors: June-October 2018. Transfusion 2019;59:3171-6.

5. Bullard JMP, Ahsanuddin AN, Perry AM, et al. The first case of locally acquired tick-borne Babesia microti infection in Canada. Can J Infect Dis Med Microbiol 2014;25:e87-9.

6. Tick-borne diseases. Manitoba: Manitoba Public Health; 2018. Available: https://www.gov.mb.ca/health/publichealth/cdc/tickborne/ (accessed 2019 Aug. 27).

7. Scott JD. First record of locally acquired human babesiosis in Canada caused by Babesia duncani: a case report. SAGE Open Med Case Rep 2017;5:2050313X17725645.

8. Krause PJ, McKay K, Gadbaw J, et al. Increasing health burden of human babesiosis in endemic sites. Am J Trop Med Hyg 2003;68:431-6.

9. Wormser GP, Lombardo G, Silverblatt F, et al. Babesiosis as a cause of fever in patients undergoing a splenectomy. Am Surg 2011;77:345-7.

10. Wormser GP, Dattwyler RJ, Shapiro ED, et al. The clinical assessment, treatment, and prevention of Lyme disease, human granulocytic anaplasmosis, and babesiosis: clinical practice guidelines by the Infectious Diseases Society of America. Clin Infect Dis 2006;43:1089-134.

11. Diuk-Wasser MA, Vannier E, Krause PJ. Coinfection by the tick-borne pathogens Babesia microti and Borrelia burgdorferi: ecological, epidemiological and clinical consequences. Trends Parasitol 2016;32:30-42.
Competing interests: None declared.

This article has been peer reviewed.

The authors have obtained patient consent.

Affiliations: Department of Internal Medicine (Yang, Battad), Section of Infectious Diseases, Department of Internal Medicine (Smith), University of Manitoba, Winnipeg, Man.

Contributors: All of the authors contributed to the conception and design of the work. Jiayu Yang drafted the manuscript. Catherine Smith and Anthony Battad revised it critically for important intellectual content. All of the authors gave final approval of the version to be published and agreed to be accountable for all aspects of the work.

Content licence: This is an Open Access article distributed in accordance with the terms of the Creative Commons Attribution (CC BY-NCND 4.0) licence, which permits use, distribution and reproduction in any medium, provided that the original publication is properly cited, the use is noncommercial (i.e., research or educational use), and no modifications or adaptations are made. See: https://creativecommons.org/ licenses/by-nc-nd/4.0/

Correspondence to: Jiayu Yang, yangj326@myumanitoba.ca

The section Cases presents brief case reports that convey clear, practical lessons. Preference is given to common presentations of important rare conditions, and important unusual presentations of common problems. Articles start with a case presentation (500 words maximum), and a discussion of the underlying condition follows (1000 words maximum). Visual elements (e.g., tables of the differential diagnosis, clinical features or diagnostic approach) are encouraged. Consent from patients for publication of their story is a necessity. See information for authors at www.cmaj.ca. 